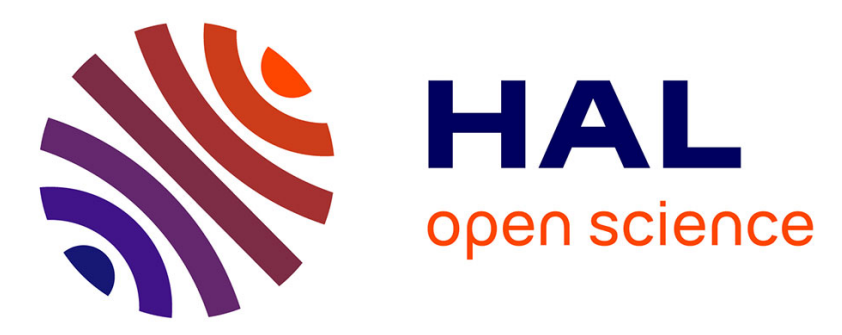

\title{
Generalized Meyer sets and Thue-Morse quasicrystals with toric internal spaces
}

Jean-Louis Verger-Gaugry, Janusz Wolny

\section{To cite this version:}

Jean-Louis Verger-Gaugry, Janusz Wolny. Generalized Meyer sets and Thue-Morse quasicrystals with toric internal spaces. Journal of Physics A: Mathematical and General J. Phys. A: Math. Gen, 1999, 32, pp.6445-6460. hal-03134994

\section{HAL Id: hal-03134994 \\ https://hal.science/hal-03134994}

Submitted on 13 Feb 2021

HAL is a multi-disciplinary open access archive for the deposit and dissemination of scientific research documents, whether they are published or not. The documents may come from teaching and research institutions in France or abroad, or from public or private research centers.
L'archive ouverte pluridisciplinaire HAL, est destinée au dépôt et à la diffusion de documents scientifiques de niveau recherche, publiés ou non, émanant des établissements d'enseignement et de recherche français ou étrangers, des laboratoires publics ou privés. 


\title{
Generalized Meyer sets and ThueMorse quasicrystals with toric internal spaces
}

\author{
J.-L. Verger-Gaugry ${ }^{\dagger}$ and J. Wolny ${ }^{\ddagger}$ \\ ${ }^{\dagger}$ Institut Fourier, University Joseph Fourier Grenoble, CNRS URA 188, BP 74- Do- \\ maine Universitaire, 38402- Saint Martin dHères, France. \\ $\$$ AGH UST - Faculty of Physics and Nuclear Techniques, University of Mining and \\ Metallurgy, al. Mickiewicza 30, 30-059 Krakow, Poland.
}

\begin{abstract}
We show that a one-dimensional aperiodic Delaunay set of points $\Lambda$ together with the Fourier transform of its autocorrelation measure (square modulus of its structure factor) at a wavevector $k=2 \pi / \lambda$, can be associated with a generalized Meyer set under some assumptions: (a) that the internal space is toric, $\mathbb{R} / \lambda \mathbb{Z}$, with a window, assumed finite, equal to the set of affine lattices of period $\lambda$ which have a non-empty intersection with $\Lambda$ and rarefaction laws at infinity, a selection rule based on a congruence mode with respect to $\lambda$; (b) a scaling exponent function, having values in $[0 ; 1]$, can be uniquely defined on the window from rarefaction laws, which is related to the scaling properties of the intensity function; (c) the projection mappings are adapted to the average lattice of $\Lambda$ and are not orthogonal. The case of Bragg peaks of the ThueMorse sequence spectrum is developed explicitly in this context.
\end{abstract}




\section{Introduction}

The notion of a model set developed by Meyer [1,2] in the context of cut-and-project schemes (CPSs), in the 1970s, has been applied successfully and extensively to the study of the structure of quasicrystals for more than ten years. Its simplicity, which is expressed by the existence of a suitable lattice in a periodization space, suitable windows in the internal space and its power for refining structure models [3] make model sets new exciting tools for studying aperiodic sets of points modelling aperiodic crystals and particularly quasicrystals.

However, the constructions of Meyer were more general. They were developed in the context of locally compact Abelian groups, that is finite-dimensional Euclidean spaces $\mathbb{R}^{m}$, compact subgroups of the infinite torus $\mathbb{T}^{\infty}$, discrete or finite groups, and so on (Rudin [12], chapter 2) and for classes of Delaunay sets greater than the class of model sets; whereas the applications to quasicrystals are only using Euclidean spaces and model sets. Here, we are interested in the fundamental question of understanding when toric internal spaces appear naturally in the study of general Delaunay point sets, for what purposes they may be used, which characteristics possess windows. For this, we assume that these Delaunay point sets still lie in a Euclidean space.

In the following, given a Delaunay set $\Lambda$, we claim that a toric internal space is important for describing the behaviour of the diffracting intensity function of $\Lambda$ and for describing its scaling exponents. We show that these scaling exponents arise from the rarefaction laws, at infinity, of distributions of points on affine lattices intersecting $\Lambda$ when some assumptions are 
satisfied. The diffracting intensity function of $\Lambda$ is expressed by the Fourier transform of the autocorrelation measure (which is the square modulus of its structure factor, normalized by the number of diffracting sites). Delaunay sets $\Lambda$ will lie on the positive real line for the sake of simplicity.

Indeed, when aperiodic point sets are no longer quasiperiodic, the search for sets supporting the components of the spectrum, Bragg, singular continuous and absolutely continuous becomes more difficult. Model sets formed by normal cut-and-project schemes with Euclidean spaces as physical space and internal space, give only pure Bragg spectra [4]. Many other possibilities could of course occur, and the full generality of the theory calls for new concepts to deal with scaling exponents associated with the singular continuous components of the spectra.

Hof [4] has developed a mathematical model of diffraction theory adapted to any Delaunay set in $\mathbb{R}^{n}, n \geqslant 1$. He shows that knowledge of the autocorrelation measure of this set is basic since it gives rise, by its Fourier transform, to the diffraction pattems of the set, known to physicists. This Fourier transform is a positive measure (Bochner's theorem). For instance, it can be computed directly by hand in the case of interpenetrating $G$-clusters, where $G$ is a finite non-crystallographic symmetry point group and when $G$-clusters are arranged quasiperiodically or not [5]. When arranged quasiperiodically in a suitable way, the system of interpenetrating $G$-clusters is a quasicrystal [6], and this approach is another view of the diffraction of the quasicrystal through the existence of the local clusters of atoms it contains.

Of importance for an arbitrary Delaunay set $\Lambda$ is the localization and the search for Bragg peaks and of peaks belonging to the singular continuous component of the spectrum of $\Lambda$. This can be carried out by a suitable Fourier-Bohr analysis [7-9], up to some limit, by the average unit cell approach $[10,11]$, typical of finite systems. Here, we propose a different scheme. We fix a certain wavevector $k$, that is a period $\lambda=2 \pi / k>0$ and want to understand the scaling properties of the intensity at $k$. We consider all the affine lattices in $\mathbb{R}$ of period $\lambda$ which have a non-empty intersection with $\Lambda$ and assume they are a finite number of them. This is expressed by the finiteness of $\Omega_{\lambda}$ (section 2), that is the fact that the window $\Omega_{\lambda}$ has a Hausdorff measure equal to zero (section 5). The second assumption is about rarefaction laws at infinity. Let $v+\lambda \mathbb{Z}$ any affine lattice intersecting $\Lambda$. If $q$ denotes the number of adjacent cells of this lattice counted from the origin, we assume that the number of points of $(v+\lambda \mathbb{Z}) \cap \Lambda$ within these $q$ cells is given by, when $q$ goes to infinity,

$$
A_{1}(q) q^{\alpha_{\lambda}(v)}+A_{2}(q) q^{d_{2}}+A_{3}(q) q^{d_{3}}+\cdots
$$

where the $A_{j}(q)$ 's are bounded, $1 \geqslant \alpha_{\lambda}(v)>d_{2}>d_{3}>\cdots \geqslant 0$. We assume a little more, that the $A_{j}(q)$ 's are all constant. We write $A_{1}(q)=\delta_{\lambda}^{\left(\alpha_{\lambda}(v)\right)}$ in section 2. Of course, all the $A_{j}(q)$ 's and the $d_{2}$ 's depend on $v$. Such an expression is called a rarefaction law of the affine lattice $v+\lambda \mathbb{Z}$ at infinity. Under these two assumptions, the $v$ 's are attributed a dominant coefficient $\alpha_{\lambda}(v)$ and are classified by lexicographical order (section 3 ) in $\left.]-\lambda / 2 ;+\lambda / 2\right]$. We claim that all $v$ 's having the maximal value of $\alpha_{\lambda}(v)$ intervene in the diffraction process when some combinations of exponentials do not cancel (theorem 1). The scaling exponent of the diffracting intensity is then directly $2 \alpha_{\lambda}(v)-1$. When some combinations of exponentials cancel, the scaling exponent becomes one of the $d_{j}$ 's, $j \geqslant 2$ (theorem 2). For this reason, we call $d_{j}$ the (fractal) levels of the scaling exponent function. The intemal space, toric, is $\mathbb{R} / \lambda \mathbb{Z}$ and the window is the finite formal external sum of affine lattices of given period $\lambda$ which intersect $\Lambda$, represented by the $v$ 's (section 5).

The structure factor of $\Lambda$ gives the Fourier transform of its autocorrelation measure. We allow a suitable Lebesgue decomposition of it according to the levels of the scaling intensity function (section 3 ) to show that the fractal scaling exponents of the intensity which appear by 
this method are corre lated to the fractal rates of occupancy at infinity of these affine lattices that intersect $\Lambda$, that is their rarefaction laws at infinity. The case of the Thue-Morse quasicrystal is presented in this context for certain values of $\lambda$. We know that the Thue-Morse sequence is not a model set since its spectrum has a non-trivial singular continuous component $[22,23]$. It is a Meyer set, a harmonious set (sections 4 and 5). Here, we investigate Bragg peaks by the present method, leaving singular continuous peaks for another contribution [21,25]. Figures showing the scaling behaviour of the diffracting intensity of the Thue-Morse sequence are reported in Wolny et al [13].

The present contribution reports (in section 5) a discussion of the axiomatics of the cutand-project scheme in order to include, for an arbitrary Delaunay set satisfying the above assumptions, a toric intemal space, scaling exponents associated with elements of windows, when windows are finite, scaling properties for the diffracting intensity. We show that the removal of one axiom is required for such a generalization of the CPS to allow more general spectra than only pure Bragg spectra. It enables one to specify the geometrical origin of the scaling exponents of the diffracting intensity. We examplify some results about the ThueMorse in this context.

\section{Definitions}

In the following, we will consider a sequence of points, as an image of a function $f$, satisfying the following assumptions:

(H). $f$ is a strictly increasing function defined on $\mathbb{N}$ taking values in $\mathbb{R}^{+}$, with $f(0)=0$. The set $\{f(n) / n \in \mathbb{N}\}$ is denoted $\Lambda$ or $\Lambda_{f}$. It represents an infinite sequence of points on the real line, satisfying the crystallographic hypothesis (Delaunay):

(D1) ( $\Lambda$ is uniformly discrete). $\exists r>0$ such that, for all $n \in \mathbb{N}$, each interval ]$-r+f(n) ;+r+f(n)[$ contains only the point $f(n)$ of the sequence,

(D2) ( $\Lambda$ is relatively dense). $\exists R>0$ such that $\forall x \geqslant 0, \exists n \in \mathbb{N}$ such that $|x-f(n)|<R$.

Let $\lambda>0$ a real number, and

$\left.\Omega_{\lambda}=\{u \in]-\lambda / 2 ;+\lambda / 2\right] / \exists n \in \mathbb{N}, f(n)=u+p \lambda$ for a certain integer $\left.p=p(n)\right\}$

the set of values of the sequence $\Lambda=\{f(n)\}$ modulo $\mathbb{Z} \lambda$. Denote

$$
\Omega_{\lambda}^{+}=\Omega_{\lambda} \cap \mathbb{R}^{+*} \quad \Omega_{\lambda}^{-}=\Omega_{\lambda} \cap \mathbb{R}^{-} .
$$

For each $u \in]-\lambda / 2 ;+\lambda / 2]$, we set

$$
\mathbb{N}_{\lambda}(u)=\{n \in \mathbb{N} / \exists p \in \mathbb{N},-p \lambda+f(n)=u\} .
$$

By definition, all the sets $\mathbb{N}_{\lambda}(u)$ are empty when $\left.\left.u \in\right]-\lambda / 2 ;+\lambda / 2\right] \backslash \Omega_{\lambda}$, and non-empty when $u \in \Omega_{\lambda}$.

Lemma 1. The following union is disjoint:

$$
\mathbb{N}=\bigsqcup_{u \in \Omega_{\lambda}} \mathbb{N}_{\lambda}(u) .
$$


This lemma gives a partitioning of the set $\mathbb{N}$ with respect to the affine lattices $u+\lambda \mathbb{Z}$, of period $\lambda$, which have a non-empty intersection with $\Lambda$.

Some of these lattices are strongly occupied by the points of $\Lambda$ and others very few. In order to understand their role in the diffraction process of $\Lambda_{f}$, we introduce their asymptotic rates of occupancy at infinity and fractional exponents. This will specify canonically the dominant terms ' $A_{1}(q) q^{\alpha_{\lambda}(v) \text { ' }}$ of their rarefaction laws at infinity.

For any $\lambda>0, q \in \mathbb{N}, N$ an integer $\geqslant 1$ and $v \in 1-\lambda / 2 ;+\lambda / 2]$, we call

$$
\mathbb{P}_{\lambda}(q)=\{n \in \mathbb{N} / f(n) \in[0 ; q \lambda]\}
$$

and

$$
\delta_{\lambda, N, q}(v)=\#\left\{n \in[0 ; N-1] \mid n \in \mathbb{N}_{\lambda}(v) \cap \mathbb{P}_{\lambda}(q)\right\} .
$$

We denote by \# $W$ or by Card $(W)$ the number of elements of an arbitrary finite set $W$. When $\lambda, q, v$ are fixed, the sequence $N \rightarrow \delta_{\lambda, N, q}(v)$ is stationary: the first value of $N$ when this sequence reaches the plateau allows one to calculate $q$ from $f$. We have

$$
f(N-1) \leqslant q \lambda<f(N)
$$

and $q=[f(N) / \lambda]$, the greatest integer less than $f(N) / \lambda$. We denote by

$$
\delta_{\lambda, q}(v)=\lim _{N \rightarrow \infty} \delta_{\lambda, N, q}(v)=\#\left(\mathbb{N}_{\lambda}(v) \cap \mathbb{P}_{\lambda}(q)\right) .
$$

For pairs $q, N$ such that equation (5) is valid, we have $\delta_{\lambda, N, q}(v)=\delta_{\lambda, q}(v)$. For each $v \in \Omega_{\lambda}$, we now consider the sets of exponents

$$
\left\{\beta \in[0 ; 1] / \liminf _{q \rightarrow \infty} \frac{\delta_{\lambda, q}(v)}{q^{\beta}}>0\right\}
$$

and

$$
\left\{\gamma \in[0 ; 1] / \limsup _{q \rightarrow \infty} \frac{\delta_{\lambda, q}(v)}{q^{\gamma}}<+\infty\right\} .
$$

Since $\mathbb{N}_{\lambda}(v) \neq \emptyset$, the first set of exponents is not empty: it contains at least $\beta=0$. We call

$$
\underline{\alpha}_{\lambda}(v)=\sup \left\{\beta \in[0 ; 1] / \liminf _{q \rightarrow \infty} \frac{\delta_{\lambda, q}(v)}{q^{\beta}}>0\right\} .
$$

Similarly, the second set of exponents above is not empty since it contains $\beta=1$ : this comes from the fact that $\#\left(\mathbb{N}_{\lambda}(v) \cap \mathbb{P}_{\lambda}(q)\right) \leqslant q$. Let us denote

$$
\bar{\alpha}_{\lambda}(v)=\inf \left\{\gamma \in[0 ; 1] / \limsup _{q \rightarrow \infty} \frac{\delta_{\lambda, q}(v)}{q^{\gamma}}<+\infty\right\} .
$$

Proposition 1. For all $v \in \Omega_{\lambda}$, we have

$$
0 \leqslant \underline{\alpha}_{\lambda}(v) \leqslant \bar{\alpha}_{\lambda}(v) \leqslant 1 .
$$

Proof. If $\underline{\alpha}_{\lambda}(v)=0$ or if $\bar{\alpha}_{\lambda}(v)=1$, the proposition is proved. Assume $\underline{\alpha}_{\lambda}(v)>0$ and $0 \leqslant \bar{\alpha}_{\lambda}(v)<\underline{\alpha}_{\lambda}(v) \leqslant 1$. Then there exist two real numbers $\beta, \beta^{\prime}$ such that

$$
\bar{\alpha}_{\lambda}(v)<\beta^{\prime}<\beta<\underline{\alpha}_{\lambda}(v) .
$$

We have $\underline{\alpha}_{\lambda}(v)>\beta-\beta^{\prime}>0$, hence $\liminf _{q \rightarrow+\infty}\left[\delta_{\lambda, q}(v) / q^{\beta-\beta^{\prime}}\right]>0$. Let us denote by $L$ this liminf. It is strictly positive and there exists a subsequence $\left\{q_{i} / i \in \mathbb{N}\right\}$ such that $\lim _{i \rightarrow+\infty}\left[\delta_{\lambda, q_{i}}(v) / q_{i}^{\beta-\beta^{\prime}}\right]=L$. Therefore, $\lim _{i \rightarrow+\infty}\left[\delta_{\lambda, q_{i}}(v) / q_{i}^{\beta}\right]=\lim _{i \rightarrow+\infty} L q_{i}^{\beta}$ 
and tends to infinity. However, $\bar{\alpha}_{\lambda}(v)<\beta$ and hence $\lim _{i \rightarrow+\infty}\left[\delta_{\lambda, q_{i}}(v) / q_{i}^{\beta}\right] \leqslant$ $\lim \sup _{q \rightarrow+\infty}\left[\delta_{\lambda, q}(v) / q^{\beta}\right]<+\infty$. A contradiction.

We will be mainly interested in rarefaction laws with the highest possible well defined fractal exponents in the dominant terms.

Definition 1. For all $v \in \Omega_{\lambda}$, we define an average sublattice in $v$ of period $\lambda$, denoted by $v+\mathbb{Z} \lambda$, of the sequence $\Lambda=\{f(n)\}$, as an affine lattice of period $\lambda$ which contains $v$ and such that

$$
\underline{\alpha}_{\lambda}(v)=\bar{\alpha}_{\lambda}(v)=1 .
$$

Definition 2. We say that $\Lambda$ satisfies assumption $\left(F_{\lambda}\right)$ when, (a) forall $v \in \Omega_{\lambda}, \underline{\alpha}_{\lambda}(v)=\bar{\alpha}_{\lambda}(v)$, and when (b) for all $v \in \Omega_{\lambda}, \lim \inf _{q \rightarrow \infty}\left[\delta_{\lambda, q}(v) / q^{\underline{\alpha}_{i}(v)}\right]$ and $\lim \sup _{q \rightarrow \infty}\left[\delta_{\lambda, q}(v) / q^{\bar{\alpha}_{\lambda}(v)}\right]$ are strictly positive, exist and are equal. In this case, we denote

$$
\alpha_{\lambda}(v)=\underline{\alpha}_{\lambda}(v)=\bar{\alpha}_{\lambda}(v)
$$

and

$$
\delta_{\lambda}^{\left(\alpha_{\lambda}(v)\right)}(v)=\liminf _{q \rightarrow \infty} \frac{\delta_{\lambda, q}(v)}{q^{\alpha_{\lambda}(v)}}=\limsup _{q \rightarrow \infty} \frac{\delta_{\lambda, q}(v)}{q^{\alpha_{\lambda}(v)}} .
$$

In particular, when

$$
\alpha_{\lambda}(v)=1
$$

we call this common limit $\delta_{\lambda}(v)$. It is by definition the average number of points of the sequence $\Lambda=\{f(n)\}$ per cell of the average sublattice $v+\mathbb{Z} \lambda$.

The notation of equation (13) is such that the quantity $\left(\alpha_{\lambda}(v)\right)$ is a superscript and not an exponent. When $\alpha_{\lambda}(v)=1$, the occupancy of the lattice $v+\mathbb{Z} \lambda$ is fairly regular. In this case, we always have $\delta_{\lambda}(v) \in[0 ; 1]$. The occupancy is fractional.

When $\underline{\alpha}_{\lambda}(v)=\bar{\alpha}_{\lambda}(v)=1$ and $\delta_{\lambda}(v)=1$, it means that we have exactly one point of the sequence, on average, congruent to $v$, per cell of the average sublattice $v+\mathbb{Z} \lambda$. This does not mean that we have a full occupancy of the lattice $v+\mathbb{Z} \lambda$. Owing to the assumption (H-D2), the number of successive cells of the lattice $v+\mathbb{Z} \lambda$ receiving no points of the sequence at all cannot be arbitrarily large.

Definition 3. A sequence $\Lambda=\{f(n)\}$ such that $\Omega_{\lambda}$ is finite and, for all $v \in \Omega_{\lambda}$,

$$
\bar{\alpha}_{\lambda}(v)<1
$$

is called singular in $\lambda$.

The terminology is reminiscent of the fact that a Bragg peak cannot exist at $k=2 \pi / \lambda$ when this condition is fulfilled, as will be shown below. result.

Conversely, for sequences of points that are subsets of lattices, we have the following

Proposition 2. If $\Lambda=\{f(n)\}$ is a subset of $\mathbb{Z} 2 r \cap \mathbb{N}, r>0$, except a finite number of elements eventually, then

(a) if $\lambda=2 r$, then $\Omega_{\lambda}=\{0\}$. The lattice $\mathbb{Z} 2 r$ is the ave rage sublattice in $v=0$ of period $2 r$ of the sequence $\{f(n)\}$, with $\delta_{\lambda}(0)=1$,

(b) if $\lambda$ is such that $\lambda / 2 r>0$ can be written as $t / w \in \mathbb{Q}$, with $t, w \in \mathbb{N} \backslash\{0\}, \operatorname{gcd}(t, w)=1$, then $\Omega_{\lambda}$ is the set of residues of $\left.\left.0,1 \times 2 r, 2 \times 2 r, \ldots,(t-1) \times 2 r \in\right]-\lambda / 2 ;+\lambda / 2\right]$ modulo $\mathbb{Z} \lambda$, and, for all $v \in \Omega_{\lambda}, \underline{\alpha}_{\lambda}(v)=\bar{\alpha}_{\lambda}(v)=1$, with $\delta_{\lambda}(v)=1 / w$, 
(c) if $\lambda$ is such that $\lambda / 2 r \notin \mathbb{Q}$, then $\Omega_{\lambda}$ is the uniformly dense set of residues of $\mathbb{Z} 2 r$ in ]$-\lambda / 2 ;+\lambda / 2]$ modulo $\mathbb{Z} \lambda$ and $\underline{\alpha}_{\lambda}(v)=\bar{\alpha}_{\lambda}(v)=0$ for all $v \in \Omega_{\lambda}$.

From case (b), we see that each time $\lambda$ is an integral multiple of $2 r$, then $w=1$, i.e. each lattice $v+\mathbb{Z} \lambda$ is an average sublattice in $v$, for all $v \in \Omega_{\lambda}$. Such collections of points are singular for any $\lambda$ which are incommensurate with the period $2 r$, from (c).

Corollary. For any Delaunay sequence $\Lambda=\{f(n)\}$, if $r_{b}$ denotes the maximal bound of $r$ such that the intervals $]-r+f(n) ;+r+f(n)[, n \in \mathbb{N}$, are mutually disjoint, then the sequence $\Lambda=\{f(n)\}$ is such that for any $\lambda<2 r_{b}$ and any $\left.\left.v \in\right]-\lambda / 2 ;+\lambda / 2\right]$, we have $\delta_{\lambda}(v) \in[0 ; 1[$ if it exists.

The value 1 cannot be reached in this case, sites being too dispersed on each affine lattice $v+\mathbb{Z} \lambda, v \in \Omega_{\lambda}$.

\section{Fourier transform decomposition by sublattices}

Let $\lambda>0$ and assume that $\Omega_{\lambda}$ is finite. Let $k=2 \pi / \lambda$ be the corresponding wavevector and $N \geqslant 1$ be an integer. We will make a Lebesgue-type decomposition of the structure factor of the set $\Lambda$, gathering diffracting sites by sublattices, setting 1 to each site as the individual scattering factor. Recall (Hof [4]) that the square modulus of the structure factor is the Fourier transform of the autocorrelation measure of $\Lambda$, normalized by the number of diffracting sites and not per unit volume. The difference between the two normalizations lies in the point density of $\Lambda$, which is assumed to be a constant. In the following, we will assume that $\Lambda$ has an average lattice, that is, that there exists $\kappa>0$ such that $\lim _{N \rightarrow+\infty} f(N) / N$ exists and is equal to $\kappa$. This means roughly that $\Lambda$ behaves as $\kappa \mathbb{Z}$. The density is then $\kappa^{-1}$. The structure factor and the diffracting intensity will arise from a Lebesgue-type decomposition of the sum $\sum_{n=0}^{N-1} 1 \mathrm{e}^{\mathrm{i} k f(n)}$. We have, from lemma 1 ,

$$
\sum_{n=0}^{N-1} 1 \mathrm{e}^{\mathrm{i} k f(n)}=\sum_{u \in \Omega_{\Lambda}}\left(\sum_{\substack{n=0 \\ n \in \mathbb{N}_{\lambda}(u)}}^{N-1} \mathrm{e}^{\mathrm{i} k f(n)}\right) .
$$

Since $k \lambda=2 \pi$, and that $f(n)-p(n) \lambda=u$ for a certain $u \in \Omega_{\lambda}$ and a certain (unique) integer $p(n)$ associated with $n$, it equals

$$
\begin{aligned}
& =\sum_{u \in \Omega_{\lambda}}\left(\sum_{\substack{n=0 \\
n \in \mathbb{N}_{\lambda}(u)}}^{N-1} \mathrm{e}^{\mathrm{i} k[f(n)-p(n) \lambda]}\right)=\sum_{u \in \Omega_{\lambda}}\left(\sum_{\substack{n=0 \\
\in \in \mathbb{N}_{\lambda}(u)}}^{N-1} \mathrm{e}^{\mathrm{i} k u}\right) \\
& =\sum_{u \in \Omega_{\lambda}} \mathrm{e}^{\mathrm{i} k t} \times \operatorname{Card}\left(\mathbb{N}_{\lambda}(u) \cap[0 ; N-1]\right) .
\end{aligned}
$$

Let us now assume that $\lambda$ is such that $\Lambda$ satisfies the assumption $\left(F_{\lambda}\right)$ with well defined rarefaction laws (equations (1), (12), (13)) for all $v \in \Omega_{\lambda}$. Then, from equations (4), (5), (13), for all large enough integer $N$, with $q=[f(N) / \lambda]$

$$
\approx \sum_{u \in \Omega_{\lambda}} \mathrm{e}^{i k u t}\left(\delta_{\lambda}^{\left(\alpha_{\lambda}(u)\right)}(u) q^{\alpha_{\lambda}(u)}+A_{2}(u) q^{d_{2}(u)}+A_{3}(u) q^{d_{3}(u)}+\cdots\right) .
$$


(Here the $d_{j}$ 's and the $A_{j}$ 's are constant, depend upon $u \in \Omega_{\lambda}$ and are indexed by $u$ only.) Since $\Lambda$ is a Delaunay set, $\lim _{N \rightarrow+\infty}[f(N) / \lambda] /(f(N) / \lambda)=1$ and since we have assumed it has a density $\kappa^{-1}$ expressed by $\lim _{N \rightarrow+\infty} N / f(N)$, then

$\approx \sum_{u \in \Omega_{\lambda}} \mathrm{e}^{\mathrm{i} k u}\left[\delta_{\lambda}^{\left(\alpha_{\lambda}(u)\right)}(u)\left(\frac{\kappa}{\lambda} N\right)^{\alpha_{\Lambda}(u)}+A_{2}(u)\left(\frac{\kappa}{\lambda} N\right)^{d_{2}(u)}+A_{3}(u)\left(\frac{\kappa}{\lambda} N\right)^{d_{3}(u)}+\cdots\right]$.

We are interested in the dominant terms. We now classify the elements $u \in \Omega_{\lambda}$ by lexicographical order in the following way: if $v$ and $w$ are any two elements of $\Omega_{\lambda}$, we say that $v>w$ if $\alpha_{\lambda}(v)>\alpha_{\lambda}(w)$, or, when $\alpha_{\lambda}(v)=\alpha_{\lambda}(w), v \leqslant w$. Therefore, there exists a stationary sequence of integers $n_{0}=1, n_{1}, \ldots$ such that

$$
v_{n_{0}=1}>v_{2}>\cdots>v_{n_{1}-1}>v_{n_{1}}>v_{n_{1}+1}>\cdots>v_{n_{2}-1}>v_{n_{2}}>v_{n_{2}+1}>\cdots
$$

with

$$
\begin{aligned}
1 \geqslant \alpha_{\lambda}\left(v_{n_{0}=1}\right) & =\alpha_{\lambda}\left(v_{2}\right)=\cdots=\alpha_{\lambda}\left(v_{n_{1}-1}\right)>\alpha_{\lambda}\left(v_{n_{1}}\right)=\alpha_{\lambda}\left(v_{n_{1}+1}\right)=\cdots \\
\cdots & =\alpha_{\lambda}\left(v_{n_{2}-1}\right)>\alpha_{\lambda}\left(v_{n_{2}}\right)=\alpha_{\lambda}\left(v_{n_{2}+1}\right)=\cdots
\end{aligned}
$$

and

$$
\begin{aligned}
& v_{1}<v_{2}<\cdots<v_{n_{1}-1} \\
& v_{n_{1}}<v_{n_{1}+1}<\cdots<v_{n_{2}-1}
\end{aligned}
$$

corresponding to the jumps of the scaling exponent function $\alpha_{\lambda}$ on $\Omega_{\lambda}$. This sequence is finite since $\Omega_{\lambda}$ is assumed to be finite. With the convention, for any integer $i \geqslant 0, \sum_{n_{i}}^{n_{i}-1}=0$, we obtain

$$
\begin{aligned}
& \approx \sum_{l=0}^{+\infty}\left[\sum_{j=n_{i}}^{n_{l+1}-1} \mathrm{e}^{\mathrm{i} k v_{j}} \delta_{\lambda}^{\left(\alpha_{\lambda}\left(v_{n_{l}}\right)\right)}\left(v_{j}\right)\left(\frac{\kappa}{\lambda}\right)^{\alpha_{\lambda}\left(v_{n_{l}}\right)}\right](N)^{\alpha_{\curlywedge}\left(v_{n_{l}}\right)} \\
&+\sum_{u \in \Omega_{\curlywedge}} \mathrm{e}^{\mathrm{i} k u}\left(A_{2}(u)\left(\frac{\kappa}{\lambda} N\right)^{d_{2}(u)}+A_{3}(u)\left(\frac{\kappa}{\lambda} N\right)^{d_{3}(u)}+\cdots\right) .
\end{aligned}
$$

(a) The case when $\min _{u \in \Omega_{\Lambda}} \alpha_{\lambda}(u)>\max _{u \in \Omega_{\Lambda}} d_{2}(u)$ : let us denote by

$$
c_{\lambda}(l)=\sum_{j=n_{l}}^{n_{l+1}-1} \mathrm{e}^{\mathrm{i} k v_{j}} \delta_{\lambda}^{\left(\alpha_{\lambda}\left(v_{n_{l}}\right)\right)}\left(v_{j}\right)\left(\frac{\kappa}{\lambda}\right)^{\alpha_{\alpha}\left(v_{n_{l}}\right)}
$$

the $l$ th coefficient. The index $l$ is called the level index of the scaling exponent. The level $l$ is constituted by $n_{l+1}-n_{l}$ elements in the window $\Omega_{\lambda}$. Denote by $l_{\text {lop }}$ the total number of levels. If $c_{\lambda}(0) \neq 0$, the intensity $I_{N}(k)$ produced by $N$ diffracting sites is scaled with $N$ as

$$
\lim _{N \rightarrow+\infty} \frac{I_{N}(k)}{N^{2 \alpha_{\lambda}\left(v_{1}\right)-1}}=\left|\sum_{j=1}^{n_{1}-1} \mathrm{e}^{\mathrm{i} k k_{j}} \delta_{\lambda}^{\left(\alpha_{\lambda}\left(\eta_{1}\right)\right)}\left(v_{j}\right)\left(\frac{\kappa}{\lambda}\right)^{\alpha_{\lambda}\left(v_{1}\right)}\right|^{2}=\left|c_{\lambda}(0)\right|^{2} .
$$

The scaling exponent $2 \alpha_{\lambda}\left(v_{1}\right)-1 \leqslant 1$ is the best one among all the possibilities. It may occur that $c_{\lambda}(0)=0$ together with a certain number of the first coefficients, hence diminishing the scaling exponent of the diffracting intensity.

Definition 4. Under the above assumptions, we say that the spectrum of $\Lambda$ has a Bragg peak at $k$ when $2 \alpha_{\lambda}\left(v_{1}\right)-1=1$ and a singular continuous peak at $k$ when $\left.2 \alpha_{\lambda}\left(v_{1}\right)-1 \in\right]-1 ; 1[$. 
Theorem 1. If there exists $1 \leqslant h<l_{\text {lop }}$, such that, for all $l=0,1, \ldots, h-1$

$$
c_{\lambda}(l)=0 \quad \text { and } \quad c_{\lambda}(h) \neq 0
$$

then

$$
\lim _{N \rightarrow+\infty} \frac{I_{N}(k)}{N^{2 \alpha_{\lambda}\left(\omega_{n_{h}}\right)-1}}=\left|\sum_{j=h_{h}}^{n_{h_{k+1}}-1} \mathrm{e}^{\mathrm{i} k v_{j}} \delta_{\lambda}^{\left(\alpha_{\lambda}\left(v_{n_{h}}\right)\right)}\left(v_{j}\right)\left(\frac{\kappa}{\lambda}\right)^{\alpha_{\lambda}\left(v_{n_{h}}\right)}\right|^{2}=\left|c_{\lambda}(h)\right|^{2} .
$$

Corollary. Under the above assumptions, the scaling exponent of the diffracting intensity, as a function of $N$, is given by

$$
2 \alpha_{\lambda}\left(v_{n_{h}}\right)-1 .
$$

In particular, a Bragg peak at $k$ can be obtained if and only if the scaling exponent is 1 , that is if and only if

$$
\alpha_{\lambda}\left(v_{n_{h}}\right)=1, \quad \text { that is for } h=0, \quad n_{0}=1 \text { and } c_{\lambda}(0) \neq 0 .
$$

In this case, the intensity of the Bragg peak at $k=2 \pi / \lambda$, per diffracting site, is

$$
\lim _{N \rightarrow+\infty} \frac{I_{N}(k)}{N}=\left(\frac{\kappa}{\lambda}\right)^{2}\left|\sum_{j=1}^{n_{1}-1} \mathrm{e}^{\mathrm{i} k v_{j}} \delta_{\lambda}\left(v_{j}\right)\right|^{2}=\left|c_{\lambda}(0)\right|^{2} .
$$

By the one-to-one mapping between $]-\lambda / 2 ;+\lambda / 2]$ and $\mathbb{R} / \lambda \mathbb{Z}$, we transport the lexicographical classification of the elements $v$ of $\Omega_{\lambda}$ and scaling exponents $\alpha_{\lambda}(v)$ to the torus. The set $\Omega_{\lambda}$ becomes a finite subset of the torus $\mathbb{R} / \lambda \mathbb{Z}$ (the internal space), viewed as a window. A scaling exponent function $\alpha_{\lambda}$ is then defined on the window which provides the scaling behaviour of the diffracting intensity. The levels are attached to the one-dimensional lattices $v+\lambda \mathbb{Z}$, that intersect $\Lambda$, and have rarefaction laws whose dominant scaling exponents are the same.

(b) The case when $\min _{u \in \Omega_{\lambda}} \alpha_{\lambda}(u) \leqslant \max _{u \in \Omega_{\lambda}} d_{2}(u)$ : denote by $l_{s e c}$ the smallest positive integer such that, for all $m \geqslant l_{\text {sec }}+1$, for all $u \in \Omega_{\lambda}, d_{m}(u)<\min _{u \in \Omega_{\Lambda}} \alpha_{\lambda}(u)$. It may occur that the secondary terms $A_{j}(u) q^{d_{j}(u)}$ of rarefaction laws becomes prominent in the scaling behaviour of the diffracting intensity just because all the above exponential sums $c_{\lambda}(l)$, which come from the primary terms, cancel or because the lexicographical order has to take into account the levels arising from the secondary exponents $d_{m}(u)$ indexed by $1 \leqslant m \leqslant l_{\text {sec }}$. We prolongate the sequence $n_{l}$ to include all these values of exponents and denote, gathering the coefficients,

$$
c_{\lambda}(l)=\sum_{j=n_{l}}^{n_{l+1}-1} \mathrm{e}^{\mathrm{i} k v_{j}} B_{j}\left(v_{j}\right)\left(\frac{\kappa}{\lambda}\right)^{b_{j}}
$$

with $B_{j}\left(v_{j}\right)=\delta_{\lambda}^{\left(\alpha_{\lambda}\left(v_{n_{l}}\right)\right)}\left(v_{j}\right)$ or $A_{p}\left(v_{g}\right)$ or the sum of both, for some $l, p \leqslant l_{s e c}$; and $b_{j}=\alpha_{\lambda}\left(v_{n_{l}}\right)$ or $d_{p}\left(v_{g}\right)$, for some $l, p \leqslant l_{\text {sec }}$. Similarly as in theorem 1 above, we have, with this new definition of the coefficients $c_{\lambda}(l)$,

Theorem 2. If there exists $1 \leqslant h<l_{\text {sec }}$, such that, for all $l=0,1, \ldots, h-1$

$$
c_{\lambda}(l)=0 \quad \text { and } \quad c_{\lambda}(h) \neq 0
$$

then

$$
\lim _{N \rightarrow+\infty} \frac{I_{N}(k)}{N^{2 b_{n_{h}}-1}}=\left|c_{\lambda}(h)\right|^{2} .
$$

It is clear that in this case, with secondary terms and exponents, we cannot obtain Bragg peaks. 


\section{Thue-Morse sequence}

One starts with the alphabet $\{a, b\}$. The set $\{a, b\}^{*}$ endowed with the operation of concatenation is the set of all finite words on this alphabet [7]. It is a free monoid generated by $\{a, b\}$. We now define the structural map (substitution rule) $\zeta$ on $\{a, b\}$ by $\zeta(a)=a b$ and $\zeta(b)=b a$. By extension, this map operates on $\{a, b\}^{*}$. We consider the infinite word $X_{\infty}$ iterated from $a$ by $\zeta$ to the right, by iterating $\zeta$. In order to generate a Delaunay set on the positive real line, we consider that we have two segments $a$ and $b$, with $a, b>0$ two real numbers, $a>b$, with the origin of the segment $a$ set at zero and the extremity of the segment $a$ at $a$ (same notation); we form $X_{\infty}$ (same notation) to the right of the segment $a$ by iterating $\zeta$ and concatenating segments, up to infinity. The points $f(n)$ will be the extremities and origins of the segments $a$ and $b$ in the infinite sequence of segments $X_{\infty}$. Set $\Lambda=\{f(n) \mid n \in \mathbb{N}\}$. We keep the same notations for the letters $a$ and $b$ and, respectively, the corresponding segments. Let $n \in \mathbb{N}$. Instead of using the substitution rule $\zeta$ for describing the infinite word $X_{\infty}$, we use an algebraic description of the successive points of the Delaunay set $\Lambda . X_{\infty}$ or $\Lambda$ is called the Thue-Morse sequence (built from $a$ ). The $n$ th-tile $t_{n}$ of the Thue-Morse sequence (for instance, [7]) is given by

$$
t_{n}=\frac{1}{2}(a+b)+\frac{1}{2}(a-b)(-1)^{S_{2}(n)}
$$

where $S_{2}(n)$ is the sum of the 2-digits in the binary expansion of $n$. In other terms, if

$$
n=a_{0}+a_{1} 2^{1}+a_{2} 2^{2}+a_{3} 2^{3}+\cdots
$$

then

$$
S_{2}(n)=a_{0}+a_{1}+a_{2}+a_{3}+\cdots
$$

each sum being obviously finite. By convention, $t_{0}=a$, that is $(-1)^{S_{2}(0)}=+1$.

The aperiodic sequence $f(n), n \in \mathbb{N}$, is given by

$$
\begin{aligned}
& f(0)=0 \\
& f(n)=\sum_{0 \leqslant m \leqslant n-1} t_{m} .
\end{aligned}
$$

Lemma 2. For all $n \geqslant 1$, we have

$$
f(n)=\frac{1}{2} n(a+b)+\frac{1}{2}(a-b) \sum_{m=0}^{n-1}(-1)^{S_{2}(m)}
$$

with

$$
\sum_{m=0}^{n-1}(-1)^{S_{2}(m)} \in\{-1,0,+1\} .
$$

Proof. If $n$ is even, the first coefficient $a_{0}$ in its binary expansion is equal to 0 . Therefore, going from $n$ to $n+1$ leads to just adding 1 to $S_{2}(n)$ to find $S_{2}(n+1)$. We have:

- if $S_{2}(n)$ is even, then $S_{2}(n+1)$ is odd,

- if $S_{2}(n)$ is odd, then $S_{2}(n+1)$ is even. 
In other terms, if $n$ is even:

$$
(-1)^{S_{2}(n)}(-1)^{S_{2}(n+1)}=-1 .
$$

Now, we prove inductively that, for any $n \in 2 \mathbb{N}$,

$$
\sum_{m=0}^{n-1}(-1)^{S_{2}(m)}=0
$$

If $n=2$, the result is true. Assume the result for $n \geqslant 2$ even. We have

$$
\sum_{m=0}^{n-1}(-1)^{S_{2}(m)}=0
$$

and

$\sum_{m=0}^{n+1}(-1)^{S_{2}(m)}=\sum_{m=0}^{n-1}(-1)^{S_{2}(m)}+(-1)^{S_{2}(n)}+(-1)^{S_{2}(n+1)}=(-1)^{S_{2}(n)}+(-1)^{S_{2}(n+1)}$

but the sum of these two quantities is zero. Hence, the result.

Proposition 3. With $\lambda=a+b$, we have $\Omega_{\lambda}=\left\{v_{1}=-b, v_{2}=0, v_{3}=+b\right\}, \alpha_{\lambda}(-b)=$ $\alpha_{\lambda}(0)=\alpha_{\lambda}(+b)=1, \delta_{\lambda}(-b)=\delta_{\lambda}(+b)=\frac{1}{2}, \delta_{\lambda}(0)=1, n_{0}=1, n_{1}=4=n_{2}=n_{3}=\cdots=$ $\operatorname{Card}\left(\Omega_{\lambda}\right)+1$.

Proof. Since, for each $n \in 2 \mathbb{N}$, we have

$$
\sum_{m=0}^{n-1}(-1)^{S_{2}(m)}=0
$$

then $\alpha_{\lambda}(0)=1, \delta_{\lambda}(0)=1$. Now denote

$$
\begin{aligned}
& \mathcal{A}^{-}=\left\{n \in 2 \mathbb{N} \mid(-1)^{S_{2}(n)}=-1\right\} \\
& \mathcal{A}^{+}=\left\{n \in 2 \mathbb{N} \mid(-1)^{S_{2}(n)}=+1\right\} .
\end{aligned}
$$

We have $2 \mathbb{N}=\mathcal{A}^{-} \cup \mathcal{A}^{+}$as a disjoint union. The injective application $\phi: x \rightarrow 2 x+2$ defined on $2 \mathbb{N}$ sends $\mathcal{A}^{-}$to $\mathcal{A}^{+}$and $\mathcal{A}^{+}$to $\mathcal{A}^{-}$. Therefore, for the distribution of points $f(n)$, with $n \in 1+2 \mathbb{N}$, on the sublattices $\pm b+\lambda \mathbb{Z}$, we have $\alpha_{\lambda}(-b)=\alpha_{\lambda}(+b)=1$ and $\delta_{\lambda}(-b)=\delta_{\lambda}(+b)=\frac{1}{2}$. Hence, $n_{0}=1$ with the other values $n_{j}$ equal to 4 , for $j \geqslant 1$.

Corollary 1. The intensity per diffracting site of the Thue-Morse sequence, at the wavevector $k=2 \pi /(a+b)$, is given by

$$
\frac{1}{4}\left|1+\cos \left(\frac{2 \pi b}{a+b}\right)\right|^{2} \text {. }
$$

Proof. From theorem 1, we have $\kappa=\frac{1}{2}(a+b)$. Hence,

$$
\lim _{N \rightarrow+\infty} \frac{\left.I_{N}(k)\right)}{N}=\left(\frac{(a+b) / 2}{a+b}\right)^{2}\left|\frac{1}{2} \mathrm{e}^{2 \mathrm{i} \pi(-b) /(a+b)}+1+\frac{1}{2} \mathrm{e}^{2 \mathrm{i} \pi(+b) /(a+b)}\right|^{2}
$$

hence the result. 
The rarefaction laws at infinity of the affine lattices $v+(a+b) \mathbb{Z}$, with $v \in \Omega_{a+b}$, are reduced to their dominant terms $\frac{1}{2} \times q^{1}$ or $1 \times q^{1}$, with scaling exponents all equal to 1 . The Thue-Morse sequence satisfies the assumption $\left(F_{\lambda}\right)$ of definition 2 for $\lambda=a+b$. The dominant scaling exponent does not change when we now consider the rarefaction laws of affine lattices $w+\frac{(a+b)}{m} \mathbb{Z}$, where $m$ is an arbitrary positive integer. It remains equal to 1 . These rarefaction laws (calculation of the coefficient $\delta_{(a+b) / m}(w)$ ) can be easily deduced from the previous ones by grouping sites according to the new periodicity $(a+b) / m$.

Corollary 2. The spectrum of the Thue-Morse sequence contains a lattice of Bragg peaks at integral multiples of $k=2 \pi /(a+b)$ and the diffracting intensity per site at $\mathrm{km}$, for $\mathrm{m}$ an arbitrary positive integer, is given by

$$
\frac{1}{4}|1+\cos (k m b)|^{2} \text {. }
$$

Proof. We just sketch the proof and refer to [21] for complete details. Let $m$ be an arbitrary positive integer and $\lambda_{m}=(a+b) / m$. We have

$$
\frac{\kappa}{\lambda_{m}}=\frac{m}{2}
$$

and, with $v \in \Omega_{\lambda_{n}}$, for $m$ odd, there exists an integer $j$ such that

$$
\lim _{q \rightarrow+\infty} \frac{\delta_{\lambda, q}\left(v-\frac{1}{2}(2 j-m+1)\right)}{q}=m \lim _{q \rightarrow+\infty} \frac{\delta_{\lambda_{n}, q}(v)}{q}
$$

for $m$ even, there exists an integer $j^{\prime}$ such that

$$
\lim _{q \rightarrow+\infty} \frac{\delta_{\lambda, q}\left(v-\frac{1}{2}\left(2 j^{\prime}-m\right)\right)}{q}=m \lim _{q \rightarrow+\infty} \frac{\delta_{\lambda_{\text {ww }}, q}(v)}{q} .
$$

Therefore, going from $\Omega_{\lambda}$ to $\Omega_{\lambda_{n}}$, leads to dividing all the coefficients $\delta_{\lambda}(v)$ by $m$. We have

$$
\lim _{N \rightarrow+\infty} \frac{I_{N}(k m)}{N}=\left(\frac{1}{2} m\right)^{2}\left|\frac{1}{2 m} \mathrm{e}^{\mathrm{i} k m(-b)}+1+\frac{1}{2 m} \mathrm{e}^{\mathrm{i} k m(+b)}\right|^{2}
$$

hence the result.

This result was also obtained by Kolar et al [18] for substitutional systems of length 2 formed with two tiles. In Gaehler and Klitzing [19], the situation is more general. Other nontrivial Bragg peaks are reported in [18], which can also be studied by the present Lebesgue decomposition of the structure factor.

We will show in another contribution $[21,25]$ that the rarefaction laws of the affine lattices of period $(p / s)(a+b)$ which intersect the Thue-Morse sequence, where $p$ and $s$ are positive integers, are given by $p$-rarefaction laws [24]. They are basically of the type

$$
A_{1}(u) q^{1}+A_{2}(q, u) q^{d_{2}(u)}
$$

where $A_{2}(q, u)$ is a bounded fractal (continuous nowhere-differentiable) function depending upon $\log N / \log 4$, where the $d_{2}(u)$ 's are all closely related to $\log p /(p-1) \log 2$. It happens that $c_{\lambda}(0)$ generally cancels for such values of periods. For instance, with $\lambda^{\prime}=3(a+b), p=$ $3, s=1, k^{\prime}=2 \pi / \lambda^{\prime}$,

$$
\begin{aligned}
\Omega_{3(a+b)} & =\{-a-2 b,-a-b,-a,-b, 0,+b,+a,+a+b,+a+2 b\} \\
& =\left(-(a+b)+\Omega_{a+b}\right) \cup\left(\Omega_{a+b}\right) \cup\left(+(a+b)+\Omega_{a+b}\right)
\end{aligned}
$$


and the sum

$$
\begin{aligned}
c_{\lambda^{\prime}}(0)=\frac{1}{6} \mathrm{e}^{2 \mathrm{i} \pi(-a-2 b) /(3(a+b))}+\frac{1}{3} \mathrm{e}^{2 \mathrm{i} \pi(-a-b) /(3(a+b))} \\
+\frac{1}{6} \mathrm{e}^{2 \mathrm{i} \pi(-a) /(3(a+b))}+\frac{1}{6} \mathrm{e}^{2 \mathrm{i} \pi(-b) /(3(a+b))}+\frac{1}{3}+\frac{1}{6} \mathrm{e}^{2 \mathrm{i} \pi(+b) /(3(a+b))} \\
+\frac{1}{6} \mathrm{e}^{2 \mathrm{i} \pi(+a) /(3(a+b))}+\frac{1}{3} \mathrm{e}^{2 \mathrm{i} \pi(+a+b) /(3(a+b))}+\frac{1}{6} \mathrm{e}^{2 \mathrm{i} \pi(+a+2 b) /(3(a+b))}=0 .
\end{aligned}
$$

This cancels the contribution of the dominant terms, extinguishing the possible Bragg peak at $k^{\prime}$. There appears a singular continuous peak at $k^{\prime}$ of well defined exponent ( $d_{2}$ exponents) with a coefficient which looks like a constant but depends fractally of $q$. The scaling exponent of the diffracting intensity is $2(\log 3 / \log 4)-1$ in this case $[21,25]$.

\section{Generalized Meyer sets}

We will slightly change the axiomatics of CPS in order to keep at the same time the Thue-Morse sequence on the real line and the relevant rarefaction laws of the affine lattices which intersect $\Lambda$. This can be formulated within the context of Meyer sets, as generalized Meyer sets under generalized cut-and-project schemes (generalized CPS), what will be explained below. First, we recall basic facts of Meyer's constructions with locally compact Abelian groups, following Meyer [1], Moody [2] and Baake and Moody [14].

Definition 5 (Classical CPS: cut-and-project scheme). Let $G$ and $H$ be two locally compact Abelian groups, and $\pi_{1}: G \times H \rightarrow G, \pi_{2}: G \times H \rightarrow H$ the canonical projections. We say that $G$ produces $H$ if there exists

(A.s) a closed subgroup $L$ of $G \times H$ satisfying:

(A.a) $L$ is discrete in $G \times H$

(A.b) $L$ is relatively dense in $G \times H$ [property $\mathrm{H}$-(D2)].

(A.c) $L \cap\{0\} \times H=\{0,0\}$ where 0 denotes the neutral element of $G$, respectively $H$.

(A.d) $\pi_{2}(L)$ is dense in $H$.

The structure of locally compact Abelian groups is well known. A locally compact group $G$ contains an open (also closed) subset $G_{1}$ of the type $\mathbb{R}^{m} \times K$ such that $K$ is a compact in the infinite torus $\mathbb{T}^{\infty}$ and that the quotient $G / G_{1}$ is a discrete group. In particular, $K$ may be a finite-dimensional torus $(\mathbb{R} / \mathbb{Z})^{l}$, for $l \geqslant 1$ any integer. To the knowledge of one of the authors, for all the applications concerning the crystallography and structure models of quasicrystals, particularly icosahedral quasicrystals and decagonal quasicrystals, only the Euclidean part was used up to now in cut-and-project schemes. Some recent results make use of $p$-adic internal spaces [15] in the spirit of the previous works of Meyer [1] and Schreiber [16], but they do not seem to be used as such by experimentalists.

The normal CPS is a collection of mappings and Euclidean spaces

$$
\mathbb{R}^{m} \stackrel{\pi_{1}}{\longleftarrow} \mathbb{R}^{m} \times \mathbb{R}^{n} \stackrel{\pi_{2}}{\longrightarrow} \mathbb{R}^{n}
$$

\section{$L$}

where $L \subset \mathbb{R}^{m} \times \mathbb{R}^{n}$ is a lattice, $\pi_{1}$ and $\pi_{2}$ the orthogonal projection mappings onto $\operatorname{Im}\left(\pi_{1}\right):=$ the physical space $=\mathbb{R}^{m}$, and $\operatorname{Im}\left(\pi_{2}\right):=$ the intemal space $=\mathbb{R}^{n} . L$ is assumed such that with $L$ the physical space produces the intemal space, $\pi_{2}(L)$ is dense in $\mathbb{R}^{n}$ and $\left.\pi_{1}\right|_{L}$ is injective. Let $\Upsilon:=\pi_{1}(L)$. The application

$$
(\cdot)^{*}=\pi_{2} \circ\left(\left.\pi_{1}\right|_{L}\right)^{-1}
$$


is well defined on $\Upsilon$ and has values in the internal space. It is extended on the $\mathbb{Q}$-span $\mathbb{Q} \Upsilon$ of $\Upsilon$. In the context of structure models of quasicrystals, we normally choose lattices $L$ which are invariant under a finite symmetry group (the icosahedral group, cyclic groups, etc) and one or several windows $[2,14]$ in the internal space $\mathbb{R}^{n}$ to select points of $L$. If $W \subset \mathbb{R}^{n}$ is a window, it satisfies the following assumptions:

W1. The window $W \subset \mathbb{R}^{n}$ is compact.

W2. $W=\overline{\operatorname{int}(W)} \neq \emptyset$.

W3. The boundary $\partial W$ of $W$ has Lebesgue measure 0 and a model set is given by

$$
\Lambda=\left\{x \in \Upsilon \mid x^{*} \in W\right\} \subset \mathbb{R}^{m} .
$$

Some properties of model sets are the following:

M1. $\Lambda$ is a Delone set [property $\mathrm{H}$ ]: it is relatively dense and uniformly discrete.

M2. $\Lambda$ is a Meyer set: $\Lambda$ is discrete and relatively dense and there exists a finite set $F$ such that $\Lambda-\Lambda \subset \Lambda+F$. Actually the class of model sets is strictly included in the class of Meyer sets.

M3. $\Lambda$ has a well defined point density $d$ (Rogers [17]), i.e. the following limit:

$$
d=\lim _{R \rightarrow+\infty} \frac{\#(\Lambda \cap B(0, R))}{\operatorname{Vol}(B(0, R))}
$$

exists, where $B(0, R)$ is the ball centred at the origin of radius $R>0$ in $\mathbb{R}^{m}$. Its volume is $\pi^{m / 2} R^{m} / \Gamma\left(\frac{1}{2}(m+2)\right)$.

M4. $\Lambda$ has a well defined spectrum composed of Bragg peaks.

We now show that the toric part $(\mathbb{R} / \mathbb{Z})^{l}$, with $l=1$, in the internal space, plays naturally a part in the representation of the Thue-Morse sequence for frequencies $\lambda$ such that $\Omega_{\lambda}$ is finite.

Lemma 3. The Thue-Morse sequence satisfies the properties MI-M3.

Proof. Clearly, M1 is satisfied for $\Lambda$. The fact that M2 is satisfied follows from lemma 2 with $F:=\{0, \pm(a-b) / 2, \pm(a-b), \pm 3(a-b) / 2\}$ since, for any $m>n \geqslant 0$, we have $f(m)-f(n)-f(m-n) \in F$. Property M3 is satisfied since

$$
\Lambda_{a v}:=\frac{1}{2}(a+b) \mathbb{Z}
$$

is the average lattice of $\Lambda$. There is one point of $\Lambda$ per cell of $\Lambda_{a v}$ and the point density $d$ of the Thue-Morse sequence is equal to $2 /(a+b)$.

Proposition 4. The Thue-Morse sequence is hamonious. 
Proof. This is a consequence of lemma 3 and theorem X in chapter II in Meyer [1].

We will analyse elsewhere characteristics of the Thue-Morse sequence, with the notions of duality following this proposition.

Now, since it is well known that the Thue-Morse sequence has a spectrum which is not only composed of Bragg peaks, but possesses a non-trivial singular continuous component (for instance, Kolar et al [18], Queffelec [8], Kakutani [22], Mahler [23]), we should remove some assumptions from the normal cut-and-project scheme in order to obtain more general spectra than Bragg spectra, as given by M4. The Thue-Morse sequence is a Meyer set which is not a model set. We know that there exists a model set which contains it strictly [2]; here, we will not use another CPS to obtain it. On the contrary, we invoke a new scheme which looks like the classical CPS. For this, we will only change the axiomatics of the CPS in a minimal way, sticking to the formalism of the previous paragraphs. We will have to join to this geometrical approach and framework the need to define simultaneously scaling exponents for the diffracting intensity function, for the singular continuous component of the spectrum. We have then to include the finite set of all lattices that intersect $\Lambda$ in a non-empty way together with their rarefaction laws, the period $\lambda$ being given.

We suggest the following scheme:

S1. Take $H:=\mathbb{R} / \lambda \mathbb{Z}$ the one-dimensional torus, as the internal space and $G:=\mathbb{R}$ the physical space.

S2. Let us denote by

$$
\pi_{\Lambda}: \frac{1}{2}(a+b) \mathbb{N} \longrightarrow \Lambda
$$

the bijective mapping from the average lattice $\Lambda_{a v} \cap \mathbb{R}^{+}$to $\Lambda$ such that, for any $n \in \mathbb{N}$,

$$
f(n)=\pi_{\Lambda}\left(\frac{1}{2}(a+b) n\right) .
$$

We have, for any integer $n$

$$
\left|\pi_{\Lambda}\left(\frac{1}{2}(a+b) n\right)-\frac{1}{2} n(a+b)\right| \leqslant \frac{1}{2}(a-b)
$$

and we denote by $\pi_{\Lambda}^{-1}$ its inverse mapping defined on the set of the elements $\{f(n) / n \in \mathbb{N}\}$, is valued in the average lattice $\Lambda_{a v}$. Call $u$ an element in $]-\lambda / 2 ;+\lambda / 2[$ and $\bar{u}$ its canonical image in $H$.

Take

$L:=\left\{(x, \bar{u}) \in G \times H \mid x \in \Lambda_{a v}, \bar{u}\right.$ such that $u \in \Omega_{\lambda}, u \equiv f\left(\frac{2 x}{a+b}\right)$ (modulo $\left.\left.\lambda \mathbb{Z}\right)\right\}$.

Lemma 4. $L$ is discrete in $G \times H$.

S3. Let us denote by $\tilde{\pi}_{\Lambda}: \mathbb{R} \rightarrow \mathbb{R}$ any strictly increasing function satisfying

$$
\left.\tilde{\pi}_{\Lambda}\right|_{\Lambda_{a r}}=\pi_{\Lambda} .
$$

We can take it to be continuous but there is no a priori reason to do so. Then we have a new CPS consisting of a collection of spaces and mappings:

$$
\mathbb{R} \stackrel{\hat{\pi}_{\hat{\Lambda} \circ \pi_{1}}}{\longrightarrow} \mathbb{R} \times \mathbb{R} / \lambda \mathbb{Z} \stackrel{\pi_{2}}{\longrightarrow} \mathbb{R} / \lambda \mathbb{Z}
$$


Lemma 5. $\tilde{\pi}_{\Lambda} \circ \pi_{1}$ is uniformly bounded with respect to $\pi_{1}$ in the sense that its restriction to L satisfies

$$
\left\|\left.\tilde{\pi}_{\Lambda} \circ \pi_{1}\right|_{L}\right\|=\sup _{z \in L}\left|\tilde{\pi}_{\Lambda} \circ \pi_{1}(z)-\pi_{1}(z)\right| \leqslant \frac{1}{2}(a-b) .
$$

We see that $\left.\left(\tilde{\pi}_{\Lambda} \circ \pi_{1}\right)\right|_{L}=\left.\left(\pi_{\Lambda} \circ \pi_{1}\right)\right|_{L}$ is injective, and that the selection mode on the closed subset $L$ is not based on a projection mode but on a congruent mode through $f$ and the period $\lambda$ which is such that $\Omega_{\lambda}$ is finite. The fact that the window $\Omega_{\lambda}$ is finite is equivalent to saying that its Hausdorff dimension in the internal space is zero. Clearly, $L$ is closed in $G \times H$, and, since we have assumed that $f(0)=0$, the properties (A.a), (A.b), (A.c) in definition 4 are satisfied. $L$ is a priori not a subgroup in $G \times H$ and assumption (A.s) has no reason to be satisfied. $\pi_{2}(L)$ is discrete in $H$ by construction. We have a ()$^{*}$ operation as in the normal CPS:

$$
(\cdot)^{*}=\pi_{2} \circ\left(\left.\left(\pi_{\Lambda} \circ \pi_{1}\right)\right|_{L}\right)^{-1}: \Lambda \rightarrow H=\mathbb{R} / \mathbb{Z} .
$$

S4. We can now choose windows as in the normal CPS: if $W$ is a window, $W$ is a subset of $\left\{\bar{u} \in \mathbb{R} / \mathbb{Z} \mid u \in \Omega_{\lambda}\right\}$. It is a compact set for which the boundary has Lebesgue measure 0 (properties W1 and W3 are satisfied). It is not the adherence of its interior, and property W2 is not satisfied.

The generalized Meyer sets we can form from the Thue-Morse sequence $f$ with respect to the frequency $\lambda$ such that $\Omega_{\lambda}$ is finite are given, similarly to the normal CPS, by

$$
\Lambda_{W}=\left\{x \in \Lambda \mid x^{*} \in W\right\} .
$$

Of course, the property M4 is no longer valid and the spectrum displays more peaks than just Bragg peaks. If the window is maximal, we obtain the full Thue-Morse sequence as defined algebraically by $f$. If the window is smaller and contains only some points inside the torus $\mathbb{R} / \lambda \mathbb{Z}$, we obtain a subset of the Thue-Morse sequence and we have only to consider, for the scaling exponent of the diffracting intensity of the reduced system of points to consider the values of the levels for the elements which are selected by the window. We have seen that the scaling exponents and the rarefaction laws (rates of occupancy at infinity) are attached to the affine lattices $v+\lambda \mathbb{Z} \in H=\mathbb{R} / \lambda \mathbb{Z}$ and can be classified according to a lexicographical order and that the dominant scaling exponent is given by equation (21).

S5. The question of whether there exists a substitute for (A.s), that is, an algebraic structure on $L$ can be partially overcome by recent results obtained by Gazeau and Miekisz [20] who have proved that there exists a canonical symmetry group on the Thue-Morse quasicrystal. By the ()$^{*}$-operation, this can be reported to the elements of the window, and globally on $L$. However, the operations of this group have no reason to be stable by classes inside the toric internal space. So, this operation is not well defined and cannot be used in this case.

\section{Acknowledgments}

Thanks are warmly expressed to Roland Bacher, Jean-Pierre Gazeau, Robert Moody and Michael Baake for very helpful discussions, inspirations and valuable comments. We would like to acknowledge the support of the Franco-Polish Polonium project 7083 that made this cooperative work possible, and the valuable comments of the anonymous referees. 


\section{References}

[1] Meyer Y 1972 Algebraic Numbers and Harmonic Analysis (Amsterdam: North-Holland)

[2] Moody R V 1997 Meyer sets and their duals The Mathematics of Long-Range Aperiodic Order ed R V Moody (Dordrecht: Kluwer) pp 403-41

[3] Hai bach T, Cervellino A and Steurer W 1998 Maximum entropy methods and quasicrystal structures Proc. Int. Conf- on Aperiodic Crystals APERIODIC '97 ed M de Boissieu, J-L Verger-Gaugry and R Currat (Singapore: World Scientific) pp 139-48

[4] Hof A 1997 Diffraction by aperiodic structures The Mathematics of Long-Range Aperiodic Order ed R V Moody (Dordrecht: Kluwer) pp 239-68

[5] Verger-Gaugry J-L 1998 Interpenetrating decagonal clusters and quasicrystals. Fourier transform and diffuse scattering. A mathematical approach Proc Int. Conf. on Aperiadic Crystals APERIODIC '97 ed M de Boissieu, J-L Verger-Gaugry and R Currat (Singapore: World Scientific) pp 29-38

[6] Cotfas $\mathrm{N}$ and Verger-Gaugry J-L $1997 \mathrm{~A}$ mathematical construction of $n$-dimensional quasicrystals starting from G-clusters J. Phys. A: Math. Gen. 30 4283-91

[7] Allouche J-P and Mendès France M 1995 Automata and automatic sequences, course 11 Beyond Quasiorystals (Les Editions de Physique) ed F Axel and D Gratias (Berlin: Springer) pp 293-367

[8] Queffelec M 1995 Spectral study of automatic and substitutive sequences, course 12 Bevond Quasicrystals (Les Editions de Physique) ed F Axel and D Gratias (Berlin: Springer) pp 369-414

[9] Bernuau G 1998 PhD Thesis Université Paris-Dauphine, France

[10] Wolny J 1998 The reference lattice concept and its application to the analysis of diffraction patterns Phil. Mag A $77395-412$

[11] Wolny J 1998 Spatial fluctuations and their influence on singular diffraction patterns Proc. Int. Conf. on Quasicrystals, ICQ6 ed T Fujiwara (Singapore: World Scientific) pp 164-7

[12] Rudin W 1962 Fourier Analysis on Groups (New York: Wiley-Interscience) ch 2

[13] Wolny J, Wnek A and Verger-Gaugry J-L 1999 Application of an average unit cell approach to diffraction analysis of Thue-Morse sequence J. Phys. Condens. Matter submitted

[14] Baake M and Moody R V 1998 Multi-component model sets and invariant densities Proc. Int. Conf on Aperiodic Crystals APERIODIC '97 ed M de Boissieu, J-L Verger-Gaugry and R Currat (Singapore: World Scientific) pp $9-20$

[15] Baake M, Moody R V and Schlottmann M 1998 Limit-(quasi-)periodic point sets as quasicrystals with p-adic internal spaces I. Phys. A: Math. Gen. 31 5755-65

[16] Schreiber J-P 1973 Approxi mations Diophantiennes et problèmes additifs dans les groupes abéliens localement compacts Bull. Soc. Math. France $101297-332$

[17] Rogers C A 1964 Packing and Covering (Cambridge: Cambridge University Press)

[18] Kolar M, Iochum B and Raymond L 1993 Structure factor of 1d systems (superlattices) based on two-letter substitution rules I. $\delta$ (Bragg) peaks J. Phys. A: Math. Gen. 26 7343-66

[19] Gachler F and Klitzing R 1997 The diffraction pattern of self-similar tilings The Mathenatics of Long-Range Aperiodic Order (Nato Series) ed R V Moody (Dordrecht: Kluwer) pp 141-74

[20] Gazeau J-P and Miekisz J 1998 A symmetry group of a Thue-Morse quasicrystal J. Phys. A: Math. Gen. 31 L435-40

[21] Verger-Gaugry J-L, Wolny J and Patera J 1999 Mathematical quasicrystals with toric internal spaces, diffraction and Thue-Morse sequence Institut Fourier Preprint 458

[22] Kakutani S 1972 Strictly ergodic symbolic dynamical systems Proc. 6th Berkeley Symp. on Mathematical Statistics and Probability vol 2 (Berkeley, CA: University of California) pp 319-26

[23] Mahler K 1927 On the translation properties of a simple class of arithmetical functions J. Math. Phys. 6 158-63

[24] Drmota M and Skalba M 1995 Sign-changes of the Thue-Morse fractal function and Dirichlet L-series Manuscripta Math. $86519-41$

[25] Verger-Gaugry J-L 1999 p-rarefaction and arithmetics of the Fourier transform of the autocorrelation measure of the Thue-Morse sequence, Institute Fourier, in preparation 